\title{
Research of Employees Psychological Capital Structure under the Background of Chinese Culture
}

\author{
Hui Qingshan ${ }^{1}$, Lou Le ${ }^{2} \&$ Cao Xuansheng ${ }^{2}$ \\ ${ }^{1}$ Research Center of Manufacturing Enterprises Management Innovation, Guangdong University of Technology, \\ Guangzhou, China \\ ${ }^{2}$ Graduate Students of Business Administration, Guangdong University of Technology, Guangzhou, China \\ Correspondence: Lou Le, Graduate Students of Business Administration, Guangdong University of Technology, \\ Guangzhou, China. E-mail: 1129057325@qq.com
}

Received: May 5, 2014

Accepted: May 26, 2014

Online Published: June 25, 2014

doi:10.5539/ibr.v7n7p175

URL: http://dx.doi.org/10.5539/ibr.v7n7p175

\begin{abstract}
Psychological capital is an individual's positive psychological state of development, which has four features and five criteria. Psychological capital structure in Chinese cultural context contains: calm, hope, optimism and confidence. Through the methods of literature research, discussion, open questionnaire, and the formal research, this paper got employees' psychological capital content structure questionnaire items under the background of Chinese culture. We collected the relevant data, used exploratory factor analysis and confirmatory factor analysis to analyze data statistically. It explored employees' psychological capital structure dimensions under the background of the Chinese culture and concluded to the psychological capital content structure questionnaire, and tested the reliability and validity of the questionnaire. Then we developed psychological capital scale that met the requirements of psychometrics, which lay the foundation for follow-up study.
\end{abstract}

Keywords: psychological capital, calm, hope, optimism, confidence

\section{Introduction}

On the basis of generalization and summarization of research achievements of positive organizational behavior, Fred Luthans (2004), Carolyn M. Youssef (2008) put forward that psychological capital structure included four aspects, namely: (1) Confidence or self-efficacy. (2) Hope (3) Optimism. (4) Resiliency. They developed psychological capital questionnaire (PCQ) consisted of 24 questions. But so far, there haven't anyone domestic who developed questionnaire of psychological capital based on the Chinese cultural background. And domestic scholar's research shows that the meaning of many problems in the field of organizational behavior under the background of Chinese culture is different from the meaning of the same problems under the background of western culture (Ling Wenquan, Fang Liluo, Zhang Zhican \& Zheng Xiaoming, 2003). It is necessary to study employees' psychological capital content structure under the background of Chinese culture.

In this paper, we first discussed the literature research, which presented some famous scholars' opinions and researching results. Then, we carried on the exploratory research and confirmatory research of the psychological capital content structure. At next, we analyzed and discussed the research results. Finally, we made a conclusion to summarize this paper.

\section{Literature Research}

Predecessors' research achievements about the psychological capital include psychological capital concept, the relationship between psychological capital and performance, etc.

Fred Luthans, James B Avery and other scholars (2006) defined psychological capital as: individual positive psychological state of development, it has four features and five criteria. The four characteristics: (1) have confidence in the challenging work and try to complete it; (2) treat the present and the future optimistically; (3) set goals and take steps to achieve goals for future; (4) be able to recover from adversity or frustration and continue until succeed. The five judgment standards include: (1) they are based on theory and research; (2) can effectively measure; (3) is relatively new and unique in the field of organizational behavior; (4) is a kind of changeable state of mind (not fixed psychological traits); (5) exerts a positive influence on work performance. On the basis of generalization and summarization of research achievements of positive organizational behavior, 
Fred Luthans (2004), Carolyn M Youssef (2008) put forward that psychological capital structure included four aspects, namely: (1) Confidence or self-efficacy. Albert Bandura defined self-efficacy as that an individual mobilize the motivation and cognitive resources in a particular context, take the necessary actions to complete a specific task. High confidence people choose challenging work and make efforts to finish it. (2) Hope. Positive psychologist C. Rick Snyder pointed out that hope is a state of motivation formed by the interaction of goals, agency and pathways. Individuals with high sense of hope think they are capable and are willing to choose the appropriate way to achieve their goals. (3) Optimism. Individual adopts the positive method to explain the intrinsic, ancient, common events, and explain outward, temporary and situational events in a negative way. Optimists treat what happens in life positively, they seldom have such negative moods as depression, guilt and remorse. (4) Resiliency. Individual's ability of getting out of adversity, failure, uncertainty and recovering from them. Restoring force includes three aspects: accept the reality, consider life as positive, adapt to environmental changes.

\section{Exploratory Research of the Psychological Capital Content Structures}

\subsection{Research Purposes}

By large-scale formal questionnaire survey, we explored the psychological capital content structure dimensions, and developed the psychological capital scale.

\subsection{Sample and Subjects}

1800 questionnaires were sent to Guangdong, Shanghai, Beijing, Henan, Guangxi, Zhejiang, Anhui, Sichuan, Chongqing, Yunnan and other provinces and cities, and 1574 effective questionnaires were taken back. The effective recovery rate was $87.4 \%$. In accordance with the principle of random, 780 samples were selected for exploratory analysis data.

\subsection{Research Tools and Statistical Methods}

This research adopts 29 questions of the beforehand test, uses Likert6 point scale, namely: completely not conform, not very conform, a bit not conform, a little conform, fairly conform, completely conform. Participants were asked to mark on the answer which conforms to their situation. Statistical methods are exploratory factor analysis, project analysis and so on, the software used is SPSS.

\subsection{The Results of the Study and Analysis}

According to the requirements of exploratory factor analysis, the relevant inspection must be conducted before the analysis, in order to check whether the data is suitable for using the factor analysis. The KMO in this study is 0.919 , it is greater than 0.5 and very significant, which indicates that the data is suitable for exploratory factor analysis.

In the exploratory factor analysis, the method of principal component analysis is used to extract factors, and the method of orthogonal varimax is used for factor rotation. Synthetically considering common degree of each item and the size of the load factor, we selected the items with high degree and large factor loading. We take the standard that eigenvalue which is greater than 1 as the intercept factor and refer to scree plot to determine the number of projects and factors. The results show that the psychological capital content structure presents four clear structure dimensions, each project's common degree is shown in table 1, the project load and dimensions are shown in table 2.

Table 1. Common degree of psychological capital content structure's constitute project $(\mathrm{N}=780)$

\begin{tabular}{ccccccccc}
\hline Item & V4 & V5 & V6 & V7 & V10 & V12 & V13 & V14 \\
\hline Common degree & 0.554 & 0.713 & 0.732 & 0.633 & 0.591 & 0.661 & 0.658 & 0.533 \\
Item & V19 & V20 & V21 & V22 & V24 & V26 & V27 & V28 \\
Common degree & 0.614 & 0.644 & 0.676 & 0.548 & 0.610 & 0.705 & 0.704 & 0.633 \\
\hline
\end{tabular}


Table 2. The factor analysis results of psychological capital content structure $(\mathrm{N}=780)$

\begin{tabular}{|c|c|c|c|c|}
\hline \multirow{2}{*}{ Project } & \multicolumn{4}{|c|}{ factor } \\
\hline & Calm & Hope & Optimism & confidence \\
\hline V6 I can overcome the bad emotion in the work, and keep stable. & 0.812 & & & \\
\hline V5 I can adjust my own negative emotions quickly in the work. & 0.797 & & & \\
\hline V7 I can face danger fearlessly in the work. & 0.726 & & & \\
\hline V4 I can keep calm in the face of difficulties. & 0.659 & & & \\
\hline V26 I have clear work goals. & & 0.771 & & \\
\hline V27 I have the patience to achieve the work objectives. & & 0.765 & & \\
\hline V28 I work with full enthusiasm. & & 0.719 & & \\
\hline V24 I am full of hope for the future work. & & 0.680 & & \\
\hline V12 In work, I can always see the good aspects. & & & 0.774 & \\
\hline V13 I think work usually produces good results. & & & 0.744 & \\
\hline V10 I always feel that the good things are more than the bad things in work. & & & 0.737 & \\
\hline V14 I always believe that we don't need to be pessimistic in work. & & & 0.556 & \\
\hline V20 I believe I can communicate with colleagues and external people well. & & & & 0.746 \\
\hline V21 I am confident in the meeting to discuss something related to my work. & & & & 0.726 \\
\hline V19 I believe I can participate in the discussion on company affairs well. & & & & 0.726 \\
\hline V22 I believe I can find good solutions to solve the problems in work. & & & & 0.610 \\
\hline Rate of variance explained $(\%)$ & 6.803 & 40.567 & 8.775 & 7.660 \\
\hline The overall rate of variance explained & & 63.80 & & \\
\hline
\end{tabular}

We integrate the meaning of various objective, refer to the related literature, and name the above four factors.

Factor 1 -- calm. It refers to keep calm mood in work, even if encountering undesirable stimulation from the outside. Topics covered include: overcome the bad emotion in the work, and keep stable; adjust my own negative emotions quickly in the work; face danger fearlessly in work; keep calm in the face of difficulties.

Factor 2 -- hope. It refers to be full of hope to work, work actively and enthusiastically and have a clear goal. It includes: have a clear job target, have the patience to achieve work objectives and work with full enthusiasm, be full of hope for the future work.

Factor 3 -- optimism. It refers to the ability to explain and think all sorts of things in work in a positive way. It includes: I can always see the good aspects in work. I think work usually produces good results; I always feel that the good things are more than the bad things in work. I always believe that we don't need to be pessimistic in work.

Factor 4 -- confidence. It refers to have confidence in work. It includes: I believe I can communicate with colleagues and external people well. I am confident in the meeting to discuss something related to my work. I believe I can participate in the discussion on company affairs well. I believe I can find the better solution to solve the problems in the work.

These four factors reflect the different aspects of psychological capital content, but it still needs to make relevant test that whether they can reflect the same topic. The results found that these four factors of psychological capital have moderate correlation, and illustrated that they measure the same topic from different aspects, the four factors together form a whole. The correlation coefficient between the factors was shown in table 3 .

Table 3. Psychological capital content 4 factor correlation coefficient matrix $(\mathrm{N}=780)$

\begin{tabular}{cccccc}
\hline & Mean value $(\mathrm{M})$ & Standard deviation $(\mathrm{SD})$ & calm & hope & opportunity \\
\hline calm & 4.446 & 0.848 & & & \\
hope & 4.633 & 0.899 & $0.514^{* * *}$ & & \\
opportunity & 4.476 & 0.873 & $0.462^{* *}$ & $0.539^{* *}$ & $0.516^{* *}$ \\
confidence & 4.526 & 0.824 & $0.532^{* *}$ & $0.572^{* *}$ & 0 \\
\hline
\end{tabular}

Note: ** shows significant at the 0.01 level. 


\section{Confirmatory Research of the Psychological Capital Content Structure}

\subsection{Research Purposes}

We use another part of the formal investigation data to test the four-factor structure model of the psychological capital in explorative study, and to examine the rationality of the structure model. At the same time, we compare the four-factor model with other competition models, and then to test the goodness of fit of the four-factor model.

\subsection{The Participants}

1574 effective questionnaires from the formal research were taken back, 780 questionnaires used for exploratory factor analysis were removed from them, then the remaining 794 were used as the samples for confirmatory factor analysis.

\subsection{Research Tools and Methods}

1) The research tools. This research adopts 29 questions of the beforehand test, uses Likert6 point scale, namely: completely not conform, not very conform, a bit not conform, a little conform, fairly conform, completely conform. Participants were asked to mark on the answer which conform to their situation.

2) Statistical analysis method. The method of confirmatory factor analysis in structural equation model and AMOS4.0 statistical software were used.

\subsection{Model Validation Results}

According to the result of exploratory factor analysis, psychological capital content structure conception model is put forward.

The data of confirmatory factor analysis were used to measure and test the proposed conceived model, and then we get the standard solution of the psychological capital content structure model, as is shown in figure 1 .

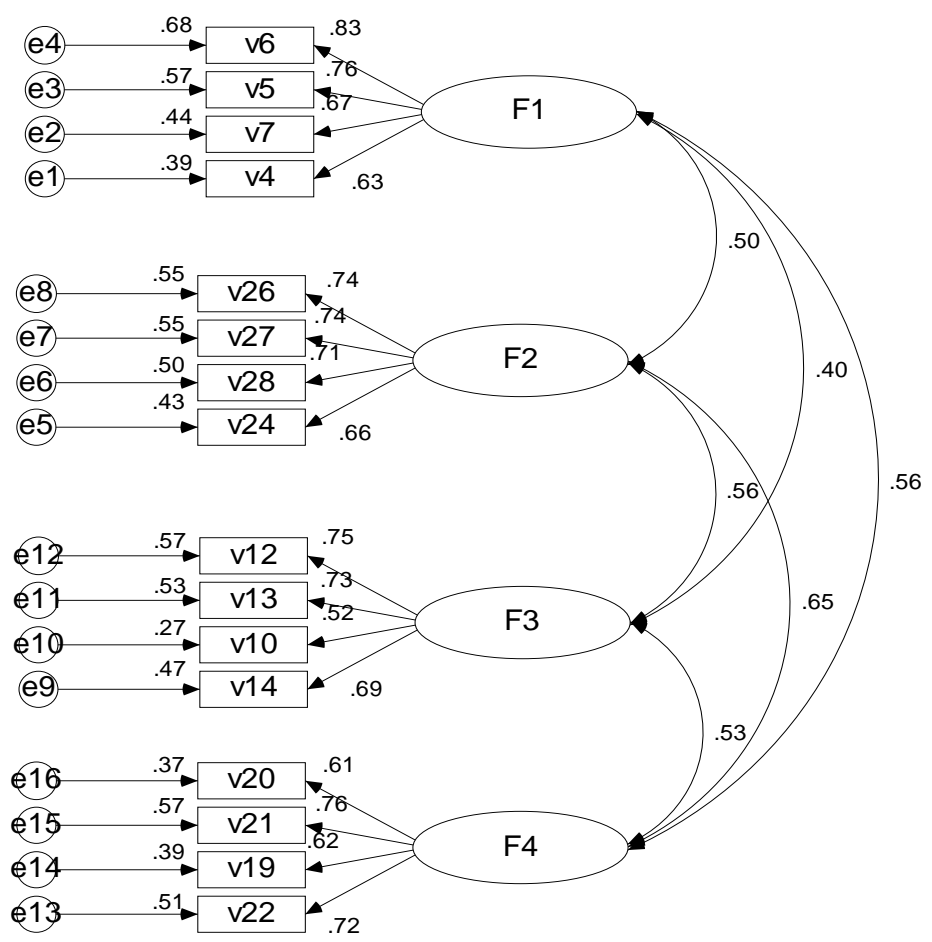

Figure 1. Complete standard solution of psychological capital content structure model

According to the theory of structural equation, we need to test the degree of fitting of the model. But here, we mainly discuss the basic fitting test and model overall fitting test.

1) Basic fitting inspection. Analysis data show that, in the four-factor model of the psychological capital content structure, the estimated parameters are all not negative, and achieved significant level; The maximum of 
absolute value of the estimated parameter statistics related to each other is 0.655 , no close to 1 ;maximum of load factor is 0.826 , the minimum value is 0.522 , both of which are between 0.5 and 0.95 ;so there has no big standard error. Therefore, it passes the basic fitting degree inspection.

2) Test of the whole fitting degree of the model. We mainly test the fitting degree of a model here, and do not involve the comparison of multiple models, therefore, we need to select indicators with judgment standard to test. So, we choose three kinds of statistic of the whole fitting degree test indicators of the model, they respectively are: the absolute fitting degree index in table 4 , the value-added fitting degree index in table 5 , and contracted fitting degree index in table 6 .

Table 4. The absolute fitting degree index of psychological capital content structure model

\begin{tabular}{cccc}
\hline GFI & AGFI & RMR & RMSEA \\
\hline 0.961 & 0.946 & 0.037 & 0.044 \\
\hline
\end{tabular}

Table 5. The value-added fitting degree index of the psychological capital content structure model

\begin{tabular}{ccccc}
\hline NFI & RFI & IFI & TLI & CFI \\
\hline 0.945 & 0.933 & 0.966 & 0.958 & 0.966 \\
\hline
\end{tabular}

Table 6. The contracted fitting degree index of psychological capital content structure model

\begin{tabular}{cccc}
\hline PGFI & PNFI & CN & NC \\
\hline 0.692 & 0.772 & 391 & 2.529 \\
\hline
\end{tabular}

From table 4, 5 and 6, we can see that the fitting degree index of the four-factor model of the psychological capital content structure all meet the demand, which shows that the four-factor model conform to the observation data, and the conceived model has been verified well.

\subsection{The Comparison of Competition Model}

Another main function of structure equation technology is to select the best matching model by the comparison of multiple models. The conceived competition models in this study include single-factor model, two-factor model, three-factor model.

1) The single-factor model. We can find from the exploratory study in front that, the four factors of the psychological capital content structure have moderate relationship, so, there may exists a single-factor model.

2) The two-factor model. We use the data from exploratory research for exploratory factor analysis, extract two factors, as a result, the V10, V12, V13, V14, V21, V22, V24, V26, V27, V28 form factor 1, V4 and V5 and V6, V7, V19, V20 form factor 2.Total variance explained is $49.341 \%$.

3) The three-factor model. By the same way, we use the data from exploratory research for exploratory factor analysis, extract three factors, as a result, V19, V20, V21, V22, V24, V26, V27, V28 form factor 1, V4 and V5 and V6,V7 form factor 2, V10, V12, V13, V14 form factor 3. Total variance explained is $57.001 \%$. The fitting index comparison between four-factor model of psychological capital content structure with single-factor model, two-factor model, three-factor model are shown in table 7.

Table 7. Comparison of fit index in the psychological capital content structure competition model fit index

\begin{tabular}{|c|c|c|c|c|c|c|c|c|c|c|c|}
\hline & \multicolumn{6}{|c|}{ Absolute fitting index } & \multicolumn{5}{|c|}{ Value-added fitting index } \\
\hline & GFI & AGFI & RMR & RMSEA & $\mathrm{NCP}$ & ECVI & NFI & RFI & IFI & TLI & CFI \\
\hline one-factor model & 0.78 & 0.712 & 0.092 & 0.127 & 1330.096 & 1.889 & 0.682 & 0.634 & 0.699 & 0.651 & 0.697 \\
\hline Two-factor model & 0.833 & 0.779 & 0.089 & 0.109 & 966.636 & 1.432 & 0.763 & 0.724 & 0.781 & 0.744 & 0.78 \\
\hline Three-factor model & 0.907 & 0.875 & 0.053 & 0.074 & 433.565 & 0.762 & 0.882 & 0.859 & 0.902 & 0.883 & 0.901 \\
\hline Four-factor model & 0.961 & 0.946 & 0.037 & 0.044 & 149.84 & 0.408 & 0.945 & 0.933 & 0.966 & 0.958 & 0.966 \\
\hline Standard for judgment & $>0.9$ & $>0.9$ & $<0.05$ & $<0.05$ & $\begin{array}{l}\text { The smaller } \\
\text { the better }\end{array}$ & $\begin{array}{c}\text { The smaller } \\
\text { the better }\end{array}$ & $>0.9$ & $>0.9$ & $>0.9$ & $>0.9$ & $>0.9$ \\
\hline
\end{tabular}


Table 7. Continue

\begin{tabular}{|c|c|c|c|c|c|c|}
\hline & \multicolumn{6}{|c|}{ Simple fitting index } \\
\hline & PGFI & PNFI & Value of $\mathrm{CN}$ & $\mathrm{X}^{2} /$ Degrees of freedom & AIC & CAIC \\
\hline one-factor model & 0.597 & 0.591 & 72 & 13.789 & 1498.096 & 1679.763 \\
\hline Two-factor model & 0.631 & 0.655 & 95 & 10.385 & 1135.636 & 1322.98 \\
\hline Three-factor model & 0.674 & 0.742 & 187 & 5.293 & 604.565 & 803.263 \\
\hline Four-factor model & 0.692 & 0.772 & 391 & 2.529 & 323.84 & 539.569 \\
\hline Standard for judgment & $>0.5$ & $>0.5$ & $>250$ & $1<\mathrm{NC}<5$ & The smaller the better & The smaller the better \\
\hline
\end{tabular}

From the comparison of 17 indexes of 3 types in Table 7, we can see that in the psychological capital content structure, the four-factor model is a more ideal one, and the other three competition models do not pass inspection in many indicators.

The results of exploratory factor analysis and confirmatory factor analysis both show that the psychological capital content structure is made up of four factors.

\subsection{Research of Reliability and Validity of the Questionnaire of the Psychological Capital Content Structure}

This research tests the reliability of psychological capital questionnaire from three aspects, namely individual item reliability, construct reliability and reliability of internal consistency.

Reliability calculation results of the three aspects show that, the coefficient of reliability of internal consistency of the questionnaire of the psychological capital content structure achieves the good level.

There are lots of indicators to measure validity. The indicators in this research include content validity, face validity, construct validity, convergent validity and criterion-related validity.

From the five aspects of validity analysis, we can judge that the questionnaire of psychological capital has good validity.

\section{Results and Discussion}

This research finds that psychological capital of employees includes four dimensions under the background of Chinese culture by exploratory factor analysis and confirmatory factor analysis, they are calm, hope, optimism and self-confidence, and every dimension is made up of four measuring subjects. The analysis of reliability and validity shows that the questionnaire of psychological capital content under the background of Chinese culture which is developed in this research has the high reliability and validity.

Fred Luthans, Carolyn M Youssef (2004) put forward that psychological capital content includes four aspects, they are self-confidence, hope, optimism and tenacity. There exists difference in two aspects between this research and Luthans' research.

First, Content structure is different. There is calm factor in this research and it doesn't exist in their research; there is tenacity factor in their research and there is not in this research. China staff has undergone many major changes in 30 years of reform and opening up, especially in the early 1990s with implementing the market economy, all departments reformed actively and transformed from the mode of planned economy to the mode of market economy operation. In the process of the transition, employees faced many challenges from the environment uncertainty, including the business philosophy transformed from production oriented to market oriented, the paternalistic management style converted into service type, authoritarian leadership style converted into democracy, the relationship between employees and units transformed from the lifelong system to the employment system, job uncertainty increased and work pressure increased and so on. Through the reform process, it may be the most important to keep calm and treat these changes with normal mind and maintain the stability of the mood. Only in this way can they adapt to the change of environment. At the same time, it is necessary to be hopeful about the future and full of enthusiasm for the work; they need to face changes optimistically as well.

Second, the same factor has different measurement projects. This research and Luthans' research have three same factors, which are self-confidence, hope and optimism, but the measurement projects of every factor is different. We use four projects to measure hope, two of which are related to the target of work, one indicates work enthusiasm, one is hopeful about the future work. Their research uses six projects to measure hope, three of which are related to the target of work, two indicate that there have many ways to solve the work problems, one 
describes the positive comments about their work. As for optimism, we use four projects to describe the meaning of treating what happened in work with positive mind from different sides. And they use six projects. To measure optimism, three of which are similar to the meaning of our projects, one indicates a kind of optimistic attitude to work and two indicate that result of work in reality is consistent with the expected. In terms of self-confidence factor, three of four projects in our research are similar to theirs. We have one project that shows confidence in face of work problems. However, in their research, one project shows confidence in solving the long-term problems, one project shows that they believe they can set work goals and one project shows they can state to a group of colleagues. Thus, even for the same item, measurement problems are also different. We think that the reasons for these differences in addition to language differences, there are differences in cultural background.

The above analysis has once again proven some research conclusions of domestic scholars, namely a lot of problems at the field of organizational behavior under the background of Chinese culture is different from those under western culture (Ling Wen-quan, Fang Li-luo, Zhang zhi-can \& Zheng xiao-ming, 2003). Therefore, foreign questionnaire of psychological capital can't be directly used to measure psychological capital of Chinese employees.

In addition, the new calm dimension in the concept of psychological capital, which is put forward in this research, is similar to the meaning of emotional intelligence. Danial Goleman (1995) put forward that emotional intelligence (EI) includes five aspects: knowing their own emotions, controlling their emotions, self-motivation, understanding others' emotions, properly handling interpersonal relationship. And Luthans (2007) think that emotion and emotional advantage is the potential psychological capital. Therefore, it can be seen that psychological capital and the concept of emotional intelligence have certain contact, but whether several other dimensions of emotional intelligence belong to the scope of psychological capital will be an issue of future research.

\section{Conclusion}

Through literature study, interview, open questionnaire, exploratory factor analysis and confirmatory factor analysis, we found that employees' psychological capital under the background of the Chinese culture is composed of four factors, namely, calm, hope, optimism, confidence.

The research of the reliability and validity of psychological capital questionnaire showed that the psychological capital questionnaire developed in this study was reliable and effective.

Although we spend a lot of time and energy on studying the employees' psychological capital content structure under the background of Chinese culture, we don't know whether the psychological capital content contains some other dimensions, as people's psychological state is complex and variable. Therefore, to explore other possible dimensions of psychological capital content is a trend of psychological capital research in the future.

\section{References}

Block, J., \& Kremau, A. M. (1996). IQ and Ego Resiliency: Conceptual and Empirical Connections and Separateness. Journal of Personality and Social Psychology, 70, 349-361. http://dx.doi.org/10.1037/0022-3514.70.2.349

Coutu, D. L. (2002). How Resilience Works. Harvard Business Review, 80, 46-55.

Erez, A., \& Isen, A. M. (2002). The Influence of Positive Affect on the Components of Expectancy Motivation. Journal of Applied Psychology, 87, 1055-1067. http://dx.doi.org/10.1037/0021-9010.87.6.1055

Fred, L., Kyle, W. L., \& Brett, C. L. (2004). Positive Psychological Capital: Beyond Human and Social Capital. Business Horizons, 47, 45-50. http://dx.doi.org/10.1016/j.bushor.2003.11.007

Kennon, M. S., \& Barbara, L. F. (2001). Psychologic Need-Satisfaction and Subjective Well-Beingwithin Social Groups. American Psychologist, 56, 25-38.

Klonhlen, E. A. (1996). Conceptual Analysis and Measurement of the Context of Ego Resiliency. Journal of Personality and Social Psychology, 70, 1067-1079. http://dx.doi.org/10.1037/0022-3514.70.5.1067

Luthans, F., Avey, J. B., Avolio, B., Norman, S. M., \& Combs, G. (2006). Psychological Capital Development: Toward a Micro-Intervention. Journal of Organizational Behavior, 27, 387-393. http://dx.doi.org/10.1002/job.373

Martin, E. P. S., \& Fausto, M. (2000). Internal Control Sense. American Psychologist, 55, 8-10.

Martin, E. P. S., \& Mihaly, C. (2000). Positive Psychology: An Introduction. American Psychologist, 55, 5-14. http://dx.doi.org/10.1037/0003-066X.55.1.5 
Rich, G. J., \& Taylor, E. (2001). Motivation and Efforts. Journal of Humanistic Psychology, 41, 21-24.

Stajkovic, A. D., \& Luthans, F. (1998). Self-Efficacy and Work-Related Performance: A Meta-Analysis. Psychological Bulletin, 124, 240-261. http://dx.doi.org/10.1037/0033-2909.124.2.240

\section{Copyrights}

Copyright for this article is retained by the author(s), with first publication rights granted to the journal.

This is an open-access article distributed under the terms and conditions of the Creative Commons Attribution license (http://creativecommons.org/licenses/by/3.0/). 